\title{
Attempts to Estimate LAI Using Airborne Flashlamps and Lasers
}

\author{
Ross Nelson, Code 923, Biospheric Sciences Branch, \\ James Kalshoven, Code 705, Sensors Development and \\ Characterization Branch, \\ David Harding, Code 921, Geodynamics Branch \\ NASA/Goddard Space Flight Center, \\ Greenbelt, Maryland 20771 \\ 301-286-9925 (voice) \\ 301-286-1757 (fax) \\ ross@Itpmail.gsfc.nasa.gov
}

\begin{abstract}
Two aircraft instruments, one a flashlamp/spectrometer - AVIS, and the other a ranging laser - SLICER, were used to collect data in conjunction with ground measurements of leaf area index. The second derivatives of the AVIS spectral reflectance curves, radius of curvature, distance ratios, and NDVIs were calculated and compared to various ground $\mathrm{LAl}$ and canopy closure measures. All relationships between AVIS spectral reflectance curve surrogates and ground LAI measures on 30 forested stands were random. SLICER estimates of average canopy height, median height, canopy-ground power return ratios, canopy power return, and other transformations of the laser data were used to model ground-measured LAI and crown closure. Statistically significant relationships were found between four laser variables and $\mathrm{LAl}$ and crown closure. However $R^{2}$ values were low, $0.17-0.38$. These linear and implicitly linear models were of little practical utility for prediction of LAI on the 24 stands considered.
\end{abstract}

\section{INTRODUCTION}

This study employs two aircraft sensors which might be used to estimate LAI or crown closure. The first is a spectrometer measuring reflective returns from vegetation illuminated by a nadir-pointing flashlamp; the second is a broad-beam profiling laser.

AVIS (Active Vegetation Index Sensor), flown aboard a helicopter, is a new GSFC instrument designed to estimate leaf area index and canopy closure [3]. The flashlamp is used to illuminate a vegetated canopy with white light in order to acquire spectral measurements between $0.46 \mu \mathrm{m}-0.84 \mu \mathrm{m}$. This portion of the spectrum includes the "red edge", the sharp rise in spectral response of green vegetation between the absorptive red wavelengths and the reflective near-infrared wavelengths. The rate of change of the slope (i.e., the second derivative) of the spectral curve, at the bottom and top of this red edge, theoretically, is related to LAI [1]. Consideration of the 2nd derivative mitigates the effects of soil background contributions.

An airborne laser recently developed by personnel at NASA has been used to characterize the vertical vegetation structure of forest canopies [2]. SLICER (Scanning Lidar Imager of Canopies by Echo Recovery) is a broad-beam system (nominally a $10 \mathrm{~m}$ spot size at target), flown aboard a T-39 Sabreliner, which measures the vertical distribution of canopy components (leaves, twigs, branches) within the cylindrical footprint of a given airborne laser pulse. This system 1) may be used to extract total tree height; 2) will usually find ground beneath a vegetated canopy; and 3) will record secondary laser pulse returns which describe subcanopy vegetation layers. These returns may be related to $\mathrm{LAl}$ and the vertical distribution of that leaf area.

The primary objective of this study is to determine if AVIS or SLICER can be used to estimate $\mathrm{LAI}$ and/or canopy closure remotely. Secondarily, as part of the AVIS analysis, the theory which suggests that the second derivative of a spectral reflectance curve is related to LAI will be tested.

\section{PROCEDURE}

II.A. AVIS Data Collection

AVIS collected flashlamp-illuminated spectral data over 28 of the 30 forested sites near the NASA/Wallops Flight Facility in northeastern Virginia in late February and 
March, 1995 (leaves-off). It collected data on nine of the 30 sites on July 25, 1995 (leaveson). All of the reflectance curves for a particular stand were averaged in order to develop a mean spectral response which was representative of the entire canopy. This average spectral response was then smoothed repeatedly using rectangular filters to remove noise from the spectral signal. Second derivatives are particularly sensitive to noise in the vegetation curve signal [1], and smoothing either the signal or the second derivative mitigates this sensitivity. Second derivative values at the following specific wavelengths were extracted from each of the stand reflectance curves for which ground data had been collected:

$0.540, \quad 0.546, \quad 0.550, \quad 0.660$, $0.670, \quad 0.680,0.690$, and $0.740 \mu \mathrm{m}$. Since second derivative maxima and minima do not always occur at the same wavelengths, the following ranges were also investigated:

minimum, $0.53-0.55 \mu \mathrm{m}$ maximum, $0.65-0.71 \mu \mathrm{m}$, maximum, $0.66-0.68 \mu \mathrm{m}$ maximum, $0.67-0.69 \mu \mathrm{m}$,

and minimum, $0.73-0.75 \mu \mathrm{m}$.

Three different LAI estimates were generated from the Licor-2000 ground estimates of LAI [5]. Needle clumping required that some adjustment be made based on the conifer component in the Licor's field of view. In addition, it was obvious from the March 1995 LAl measurements that light occlusion by stem boles and branches contributed significantly to the LAl estimates. Hardwood stands with no leaf area registered LAls of 0.4 to 1.0. Three different estimates of LAl were generated for each stand from the Licor-2000 data. The first, $L$, was the unaltered Licor-2000 LAl estimate. The second, $L_{1}$, incremented this $L_{A l}$ estimate for needle clumping based on the proportion of conifer in the stand; and the third, $L_{2}$, accounted for both needle clumping and hardwood bole/branch occlusion. Two canopy density measurements were considered, a Licor-2000 estimate - $\mathrm{CCL}$, and a second measured using a spherical densiometer - $c_{s}$ [4]. The three LAI and two canopy density estimates were compared to the various AVIS data transformations. Unadjusted $L A I$ values ranged from 0 to 5 , adjusted LAI values from 0 to 8 . Canopy closures ranged from $0-100 \%$.

II.B. SLICER Data Collection
Airborne laser data were acquired by a profiling version of the SLICER instrument on October 7, 1993. The NASA T-39 overflew mixed conifer/hardwood forests of the Delmarva Penninsula and Maryland. Twenty-four forest stands measured by the laser were identified using aircraft GPS/INS information, VHS videography acquired coincidently with the laser data, and road maps. It was estimated that ground samples were located within $50 \mathrm{~m}$ of the actual laser transect. The SLICER waveform data were processed to identify the ground return and canopy returns from each transmitted laser pulse within the stands [2].

The following laser variables were developed from the waveform data:

- total tree height: range to peak ground return minus range to the top of the tree.

- canopy amplitude: integrated pulse power returned by the canopy.

- canopy amplitude ratio: $\sum$ canopy return/( canopy+ + ground return).

- canopy area: the largest cumulative canopy return prior to normalization.

- median canopy height: that height at which half the canopy lies above and half below.

- nonzero bins (\%): the percentage of vertical bins which registered laser light returns, indicating the presence of vegetation.

- canopy thickness: (range to median canopy height minus range to tree top)* 2 ; i.e., twice the thickness of the top portion of the canopy which contained $50 \%$ of the leaf surface area.

These SLICER variables were compared to ground-measured LAI and canopy closure.

The 24 forest stands were measured using two LAl-2000s [5] on October 17, 18, and 19, 1995, two years after the laser overflight. Thirty below-canopy observations were acquired along a single transect approximately $150 \mathrm{~m}$ long in each stand. This ground transect was located along what was thought to be the ground trace of the airborne laser. Two LAI measurements, $L$ and $L_{1}$ (described in Section II.A), were calculated, as was one canopy closure estimate, CCL. Spherical densiometer measurements were not taken since all 24 canopies exhibited closures of over $90 \%$. No Licor measurements were acquired leaves-off, therefore no $L_{2}$ estimates were calculated. LAI measurements (L) ranged from 2 to 5 ; $L_{1}$ estimates ranged from 2 to 6 . 
III.A. AVIS Analysis

\section{RESULTS}

Scatterplots were produced in order to determine if simple predictive relationships existed between the March and August AVIS and ground LAl and canopy closure estimates. In all, 120 scatterplots were generated, i.e., 12 AVIS wavelength or wavelength range 2 nd derivatives $x$ (3 LAI + 2 cc estimates) $x$ (March obs. only or March and August obs.). Without exception, a random scatter of points was depicted in each; no relational patterns were noted. Stratification by date or consideration of only those stands which were predominantly hardwood or coniferous did not improve the results. Attempts to estimate changes in LAl between March and August were equally unproductive. The high spectral resolution data acquired under conditions of nadir illumination could not be used to predict LAl on these conifer/hardwood sites.

III.B. SLICER Analysis

The seven SLICER variables were compared to estimates of LAl in 24 forested stands. The SLICER measurements were an average of 13 to 15 pulses per plot. These are compared to 30 LAl measurements per stand. There are locational inaccuracies in these data, but attempts were made to mitigate these inaccuracies by selecting relatively large, homogeneous forest targets.

Four of the seven SLICER variables exhibited some level of correlation to the three ground reference metrics, $L, L 1$, and $c c L$. At best, all relationships have a high degree of scatter, as quantified by the highest $R^{2}$ value of 0.38 for the relationship between canopy amplitude ratio and $\mathrm{CCL}$. The four SLICER variables most useful for predicting $L$ and $C C L$ were the canopy amplitude ratio, canopy area, median height, and canopy amplitude. Though all four variables explained significant variability in ground-measured LAI and canopy closure estimates, $R^{2}$ estimates were low (range: 0.17 0.38 ), and the univariate relationships serve no practical utility.

The calculation of $L_{1}$ values and consideration of alternate models, including the use of natural log models to control variability, did not improve regression performance. Results indicate that, on the 24 stands considered in this study, SLICER variables were weakly related to ground estimates of LAI and canopy closure. However the natural variability of the 24 stands and the locational inaccuracies inherent in the registration process precluded the use of these laser variables for $\mathrm{LAl}$ and $\mathrm{CCL}$ prediction.

\section{CONCLUSIONS}

Neither AVIS nor SLICER data proved to be useful for predicting LAl or canopy closure as measured using an LAl-2000 and spherical densiometer. Second derivative values of the AVIS spectral curves in the vicinity of the top of the green hump and the bottom and top of the red edge were unrelated to LAl and canopy closure estimates. Though elegant in theory, second derivatives are sensitive to noise. The variability inherent in forest stands and inherent in the measurement of those stands made the application of that theory for LAI determination problematic on the 30 stands considered in this study. Weak univariate relationships were noted between SLICER measurements and ground-measured estimates of LAI and canopy closure. The airborne laser measured forest height and the vertical distribution of foliage within the canopy. Seven variables were developed in an attempt to consolidate the information in the ranging data; none of the seven considered could be used in any practical fashion to predict LAI or canopy closure on 24 forested sites in Maryland.

\section{REFERENCES}

1. Hall, F.G., K.F. Huemmrich, and S.N. Goward. 1990. Use of Narrow-Band Spectra to Estimate the Fraction of Absorbed Photosynthetically Active Radiation. Remote Sens. of Environ. 32(1): $\quad 47-54$

2. Harding, D.J., J.B. Blair, J.B. Garvin, and W.T. Lawrence. 1994. Laser Altimetry Waveform Measurement of Vegetation Canopy Structure. Proc., IGARSS '94, Pasadena, CA. Vol II: 1251-1253.

3. Kalshoven, J.E. 1996. Airborne ground illuminator for hyperspectral validation and calibration. Proc., SPIE, August 5-6, Denver, Colorado. Vol. 2820: 67-71.

4. Lemmon, P.E. 1957. A New Instrument for Measuring Forest Overstory Density. Jour. of Forestry 55(9): $\quad$ 667-669.

5. Licor. 1992. LAI-2000 Plant Canopy Analyzer. Operating Manual., Licor, Inc., Lincoln, Nebraska. 